\title{
Association of tumour microRNA profiling with outcomes in patients with advanced urothelial carcinoma receiving first-line platinum-based chemotherapy
}

Joaquim Bellmunt ${ }^{\star}, 1,2,3$, Chensheng Willa Zhou ${ }^{1}$, Stephanie A Mullane ${ }^{1}$, Lillian Werner ${ }^{1}$, Mary-Ellen Taplin ${ }^{1,3}$, André P Fay ${ }^{1}$, Toni K Choueiri ${ }^{1,2,3}$, Anna Orsola ${ }^{1}$, David Y Takeda ${ }^{1,2,3}$, William C Hahn ${ }^{1,2,3,4}$, Jaegil Kim ${ }^{4}$, Guru Sonpavde ${ }^{5}$ and Michaela Bowden ${ }^{1}$

${ }^{1}$ Departement of Medical Oncology, Dana-Farber Cancer Institute, 450 Brookline Avenue, Boston, MA 02215, USA; ${ }^{2}$ Departement of Medical Oncology, Brigham and Women's Cancer Center, Brigham and Women's Cancer Center, Boston, MA 02115, USA; ${ }^{3}$ Departement of Medical Oncology, Harvard Medical School, 25 Shattuck Street, Boston, MA 02115, USA; ${ }^{4}$ The Eli and Edythe L. Broad Institute, 415 Main Street, Cambridge, MA 02142, USA and ${ }^{5}$ Departement of Medical Oncology, University of Alabama at Birmingham (UAB), 1720 2nd Avenue S, Birmingham, AL 35233, USA

Background: Tumour expression of selected microRNAs (miRs) correlates with cisplatin efficacy in multiple cancers. We investigated the role of selected miRs in patients receiving cisplatin-based therapy for advanced urothelial carcinoma (UC).

Methods: RNA was extracted from formalin-fixed paraffin-embedded tumour from 83 advanced UC patients who received cisplatin. A miR panel based on relevance for platinum sensitivity and UC was studied by quantitative reverse transcription quantitative PCR (RT-qPCR). Association of progression-free survival (PFS) with miR expression was analysed using cox regression. Selected TFs were chosen by association with the panel of miRs using the Transcription Regulation algorithm (GeneGo MetaCore + MetaDrug version 6.23 build 67496). Bladder cancer (BC) cell lines were used to investigate the previously described role of miR-21 mediating cisplatin sensitivity.

Results: The 83 patients had a median PFS of 8 months. In multivariate analysis, higher levels of E2F1 ( $P=0.01$, HR: $1.95(1.14,3.33))$, miR-21 $(P=0.01, \operatorname{HR}: 2.01(1.17,3.45))$ and miR-372 $(P=0.05, \mathrm{HR}: 1.70(1.00,2.89))$ were associated with a shorter PFS. In the $8 \mathrm{BC}$ cell lines, miR-21 was not shown to be necessary nor sufficient for modulating cisplatin sensitivity.

Conclusions: In metastatic UC patients treated with cisplatin-based therapy, high primary tumour levels of E2F1, miR-21 and miR372 are associated with poor PFS independent of clinical prognostic factors. The in vitro study could not confirm miR-21 levels role in modulating platinum sensitivity.

In 2015, more than 72000 new cases of urothelial carcinoma (UC) were expected to be diagnosed in the United States, with $30 \%$ of initial cases presenting with muscle-invasive disease (Siegel et al, 2015). Metastatic UC remains incurable in the vast majority of patients, with a median progression-free survival (PFS) and overall survival (OS) of $\sim 8$ and 14 months, respectively, with front-line cisplatin-based chemotherapy (von der Maase et al, 2005; Sonpavde and Bellmunt, 2015). Cisplatin-based chemotherapy induces an overall response rate of $\sim 50 \%$, with select patients obtaining sustained responses (Bajorin et al, 1999; Apolo et al,

*Correspondence: Dr J Bellmunt; E-mail: joaquim_bellmunt@dfci.harvard.edu

Received 2 December 2015; revised 27 April 2016; accepted 29 April 2016; published online 23 June 2016

(c) 2016 Cancer Research UK. All rights reserved 0007-0920/16 
2013). In an updated report of the randomised trial comparing methotrexate, vinblastine, doxorubicin and cisplatin (MVAC) with gemcitabine and cisplatin, survival at 5 years were $15.3 \%$ and $13 \%$, respectively (von der Maase et al, 2005). Identifying biological factors predicting chemotherapy benefit would spare non-responders the toxic effects of chemotherapy, and direct them towards novel therapies. Therefore, it is imperative to determine new biomarkers to predict which patients demonstrate durable benefits from platinum therapy.

MicroRNAs (miRs) are small, single-stranded non-coding RNAs, which function as oncogenes or tumour suppressors by exerting critical roles in RNA silencing and post-transcriptional gene expression regulation (Guo et al, 2010; Bartel, 2004). They are usually 21-55 nt and single-stranded RNAs. miRs are located in intragenic and intergenic regions of the genome and can be transcribed separately or with their host genes. The miR/RNAinduced silencing complex connects to an mRNA target to degrade the mRNA or to prevent translation of the mRNA. Hence, miRNAs are key regulators and can modulate many biological processes. Moreover, given their small size, miRs are likely to be better preserved (compared with proteins and mRNA) in formalin-fixed paraffin-embedded (FFPE) tissue and comparable to expression in fresh frozen tissue (Dijkstra et al, 2012). miRNA expression has been shown to be altered in UC and shown to correlate with response to platinum drugs in multiple other malignancies such as non-small-cell lung cancer, ovarian cancer and bladder cancer (Yu et al, 2008; Zhang et al, 2008; Dyrskjot et al, 2009; Guancial et al, 2014). In addition, transcription factors (TFs) have critical upstream roles in upregulating genes mediating carcinogenesis and therapy response (Wushou et al, 2014; Lee et al, 2015; Mukherjee et al, 2015). Thus, both miRs and TFs modify gene expression downstream and upstream from the genes, respectively. However, understanding the roles of these molecules in modifying UC biology is in its infancy.

We hypothesised that a panel of miRs and TFs expressed in UC tumour tissue may be associated with durable PFS in patients receiving platinum therapy for metastatic UC.

We analysed archival FFPE tumour tissue derived from 83 patients treated with first-line platinum therapy for metastatic UC through a time to event analysis and by examining extreme responders within the cohort. We then tested the association of miRs identified to be significant with sensitivity to cisplatin in a panel of UC cell lines.

\section{MATERIALS AND METHODS}

Patients and tissue specimens. All tissue samples from patients were collected at the time of biopsy by transurethral resection of the bladder tumour or radical cystectomy (RC). A component of transitional cell carcinoma was required. All patients were required to have subsequently received first-line platinum-based chemotherapy for locally advanced or metastatic UC. Informed consent was obtained from each patient and institutional review board approval was obtained from Dana-Farber Cancer Institute before data acquisition and genetic analysis. Baseline clinicopathological characteristics, prognostic factors, response to chemotherapy and clinical follow-up were retrospectively collected.

RNA extraction. The H\&E stains were prepared on $4-\mu \mathrm{M}$ sections from the FFPE tissue blocks of tumours from all patients and a subset of normal bladders. Pathology review was undertaken to identify the percentage cellularity of the tumour, the size of the tumour region of interest and the presence of benign cells. A cutoff of 70\% tumour cellularity was the benchmark for subsequent RNA extraction and downstream reverse transcriptase (RT)-PCR. The AllPrep DNA/RNA FFPE Kit (Qiagen, Germantown,
MD, USA) was utilised for the RNA extraction from FFPE tumours (see in vitro section below for cell-lines studies). Purified RNA was quantified utilising the Quant-iT RiboGreen assay (Life Technologies, Carlsbad, CA, USA) over the concentration range $1-100 \mathrm{ng} \mu \mathrm{l}^{-1}$.

miR and TF selection. We completed a Pubmed literature search to identify the most frequently cited miRs associated with the terms bladder, cancer, and metastasis and cisplatin treatment (Supplementary Table 1). MicroRNAs were only included if three or more references were found to support its inclusion. In order to determine what TFs may be associated with the panel of miRs, we used the biological networks tool using the Transcription Regulation algorithm (GeneGo MetaCore + MetaDrug version 6.23 build 67496, Thompson Reuters, Life Sciences Research; https://portal.genego.com/). The miRTarBase (Release 6.0 September 2015) was utilised to assess the miRNA-target interactions that are experimentally validated. The E2F1 interaction with hsa-miR21-5p has been validated experimentally by reporter assay, western blot and microarray, which together are characterised as strong evidence of an interaction. We ran confirmatory analysis using miRTarBase (Release 6.0 September 2015).

RT-PCR to assess miR and TF levels. For miR expression levels, we utilised $100 \mathrm{ng}$ of RNA in each RT reaction with the TaqMan MicroRNA reverse transcription kit (Life Technologies) and subsequent PCR with the TaqMan Universal PCR Master Mix (Life Technologies), according to the manufacturer's protocol. Target-specific stem-loop precursor probes (see Supplementary Table 2 for probe details) were utilised in the RT step instead of random hexomers. Single-plex assays were set up for the 14 miRs including RNU48, which was utilised as the endogenous small RNA reference control.

For TF expression levels (selected based on network analysis using miRNA results - see below in statistical analysis section), we utilised 200 ng RNA input into $20 \mu \mathrm{l}$ reverse transcription reaction (High-Capacity cDNA Reverse Transcription Kit, Life Technologies), and $1 \mu \mathrm{l}$ of the subsequent cDNA product was transferred to the Taqman PCR assay (Life Technologies). GAPDH was utilised as the reference housekeeping gene.

Real-time PCR was performed on Applied Biosystems 7900HT (Life Technologies, Thermo Fisher Scientific corporation, Foster City, CA, USA; https://www.thermofisher.com) using standard protocol. Results were analysed using the $\Delta \Delta \mathrm{Ct}$ or comparative $\mathrm{Ct}$ method. Ct values were calculated from the real-time amplification curves, where the threshold was set at 0.02 .

Statistical and bioinformatics analysis. Expression levels of TFs and miRs were summarised using median and interquartile ranges. $\mathrm{TF}$ and miR levels were dichotomised at the median.

Progression-free survival was defined as time from the start day of treatment for metastatic disease to the date of progression or death, whichever occurred first. Patients alive without progression on the last date of follow-up were censored. For time to event analysis, Kaplan-Meier estimate was used to summarise PFS. Progression-free survival was considered as the best estimate because it captures more directly the benefit of chemotherapy while OS is affected by non-disease-related death (toxicity, comorbid conditions and others). An exploratory analysis of OS was performed despite the limitation in the sample size and in the number of events.

Cox regression model was used to assess the association of TF and miR levels with PFS in both univariate analysis and multivariate analysis, adjusting for Eastern Cooperative Oncology Group (ECOG)-performance status (PS) and visceral metastasis. The HR and 95\% CI are provided as well. Likewise, treatment type (M-VAC vs Cisplatin Gemcitabine) was also included as a covariate in the analysis. 
Patients were then divided into pre-defined extreme outlier groups based on PFS outcomes, where alive without progression at 12 months was considered good outcome and death or progressive disease (PD) within 4 months poor outcome. Four months was chosen as the cutoff to exclude patients that received less than two cycles and to capture patients assessed for disease response that showed PD at first evaluation. Patients with non-evaluable disease, and patients who went off therapy due to toxicity were removed from the analysis. Wilcoxon's rank sum test was used to assess association of $\mathrm{miR}$ and TF levels between the extreme responder groups. An exploratory outcome analysis of miR-21, E2F1, EZH2 and SUZ12 expression in the TCGA was undergone (Cancer Genome Atlas Research Network, 2014), as well as the association of these markers with the molecular subtypes.

In vitro testing. The activity of cisplatin was evaluated in eight UC cell lines to generate a dose-response curve and calculate the concentration that inhibits growth by 50\% (GI50): 647V, J82, RT112, RT4, T24, TCC-SUP, UMUC3 and VMCUB1. Cells were plated in 96-well plates at a density of 2500 cells per well and allowed to adhere. Cells were subsequently treated with cisplatin at indicated concentrations using phosphate-buffered saline as the vehicle. Cells were grown for $72 \mathrm{~h}$ and cell viability assessed by Cell Titer Glo (Promega, Promega Corporation, Madison, WI, USA) according to the manufacturer's guidelines using an Envision plate reader. The GI50 was calculated by nonlinear regression using Graphpad Prism (GraphPad Software, Inc., La Jolla, CA, USA). Results represent the average and s.d. of at least three independent experiments done in triplicate.

RNA was isolated using Qiagen miRNeasy Mini kit, and specific miRs reverse transcribed using Applied Biosystems Taqman MicroRNA Reverse Transcription kit and Gene Expression Assay. Real-time PCR was performed using Applied Biosystems Taqman Universal Master Mix II and run on ABI Quantstudio 6. The level of miR-21 was normalised to the average of RNU44 and RNU48 snoRNAs.

To further evaluate the role of miR-21 in cisplatin sensitivity, we measured drug sensitivity after upregulation of miR-21 in the cell lines with the lowest expression levels of miR-21, or suppression of miR-21 in the lines with the highest expression of miR-21. Expression of precursor miR-21 (Genecopoeia, Rockville, MD, USA) by lentiviral transduction resulted in increased levels of miR21 as measured by qRT-PCR relative to transduction with control precursor miRNA. The effect of miR-21 suppression was assessed by transduction with a lentivirus expressing antisense to miR-21 (Genecopoeia) or control. Viruses were generated by co-transfection into 293T cells with pVsVg and pDelta 8.9 using Transit-LT1 transfection reagent (Mirus Bio., Genecopoeia), and virus collected $48 \mathrm{~h}$ after transfection. Infections were done in the presence of $4 \mu \mathrm{g} \mathrm{ml}^{-1}$ polybrene and selected with either puromycin or hygromycin. As antisense suppression of miR-21 does not necessarily result in its degradation, we confirmed suppression by a miR-21 reporter assay. Reporter construct for miR-21 was purchased from Active Motif (Carlsbad, CA, USA), which expresses luciferase with miR-21-binding sites in the $3^{\prime}$-UTR. A luciferase vector that lacks the $3^{\prime}$-UTR was used as a control. Transfections were done in 96-well format using Lipofectamine 2000 (Thermo Fisher Scientific corporation). Cells were collected $48 \mathrm{~h}$ after transfection and luciferase activity measured using Active Motif Lightswitch Luciferase Assay kit. Results represent the average and s.d. of three independent replicates.

\section{RESULTS}

Patient characteristics. We identified FFPE primary tumours of 83 patients who received first-line cisplatin-based chemotherapy
Table 1. Patient characteristics

N

Clinical stage at $\mathrm{dx}$ of muscle invasive disease

$\mathrm{T} 1$

T2-T3a

$\mathrm{T} 3 \mathrm{~b}$

T4

Any $\mathrm{T}, \mathrm{N}+$

Any $\mathrm{T}$, any $\mathrm{M}, \mathrm{M}+$

Unknown

\begin{tabular}{l|r}
1 & 11 \\
9 & 11
\end{tabular}

Regimen

\begin{tabular}{|l|r}
\hline GC & 32 \\
MVAC & 45
\end{tabular}

Unknown

ECOG-PS

\begin{tabular}{|l|r|r|}
\hline 0 & 46 & 55 \\
1 & 32 & 39 \\
Unknown & 5 & 6 \\
\hline
\end{tabular}

\section{Visceral disease}

\begin{tabular}{|l|r|r}
\hline No & 44 & 53 \\
Yes & 33 & 40 \\
Unknown & 6 & 7
\end{tabular}

\section{Response}

CR
PR

PR

PD

Unknown

\begin{tabular}{l|l}
8 & 10
\end{tabular}

PFS

\section{$<4$ months}

>9-12 months

8
37
11

11

8
19

\begin{tabular}{r|r}
32 & 39 \\
45 & 54 \\
6 & 7
\end{tabular}

6

54

Abbreviations: $\mathrm{CR}=$ complete response; $\mathrm{dx}=$ diagnosis; $\mathrm{ECOG}-\mathrm{PS}=$ Eastern Cooperative Oncology Group-performance status; GC=gemcitabine and cisplatin; MVAC = methotrexate, vinblastine, doxorubicin and cisplatin; PFS = progression free survival; $P R=$ partial response; $\mathrm{SD}=$ stable disease.

(Table 1) for metastatic UC, including patients with N1 disease. The median PFS was 12 months and 43 patients experienced objective disease progression or died.

Selection of miRNA for tumour tissue profiling. In silico data mining of the literature identified a panel of 14 miRs: miR-21; miR-106b; miR-10b; miR-146a; miR-146b; miR-371; miR-372; miR-373; miR-1224; miR-1248; miR-200c; Let-7i; miR-27a; and miR-26b (Supplementary Table 2).

RT-PCR for miRs and TFs in tumour tissue. We performed the TaqMan-based real-time RT-PCR analysis to examine expression levels of the 14 selected miRs in total RNA extracted from the primary FFPE tumours of all 83 patients and 8 normal bladder samples. Median dCt values and interquartile range were calculated for each miR (Supplementary Table 3). miR-200c (5.95 (4.87, $6.87)$ ) was the highest and miR-10b $(-1.22(-2.02,0.005))$ the lowest expressed $\mathrm{miR}$ in the tumour tissue. Tumour expression of TWIST1, FOXO3, RELA, E2F1 and SMAD4 was also measured in the tumours. Median dCT values and interquartile range were calculated for each TF (Supplementary Table 4).

Selection of miRNA and TFs for tumour tissue profiling. E2F1, TWIST and RELA were selected due to their clinical significance in bladder transitional cell carcinoma (Wushou et al, 2014; Lee et al, 2015; Mukherjee et al, 2015). All 3 were found in the top 20 statistically significant $(P<0.05)$ TFs associated with the original panel of 14 miRs with GScores $>100$, utilising the Transcription Regulation algorithm (GeneGo MetaCore, Thompson Reuters, Life Sciences Research) with default settings.

Association of miR and TF tumour tissue expression with PFS and OS. We next analysed the differential expression levels 
Table 2. Association of miR-21, miR-372 and E2F1 with PFS in univariate and multivariable analyses

\begin{tabular}{|c|c|c|c|c|c|c|c|}
\hline & $N$ & $\begin{array}{c}\text { Progression } \\
\text { or death }\end{array}$ & $\begin{array}{c}\text { Median PFS } \\
\text { (months) }\end{array}$ & $P$-value & $\mathrm{HR}$ and $95 \% \mathrm{Cl}$ & $P$-value & $\mathrm{HR}$ and $95 \% \mathrm{Cl}$ \\
\hline miR-21 & & & & 0.009 & & 0.01 & \\
\hline$\leqslant 0.10$ & 41 & 20 & 26 & & 1.00 (reference) & & 1.00 (reference) \\
\hline$>0.10$ & 39 & 23 & 8 & & $1.97(1.18,3.30)$ & & $2.01(1.17,3.45)$ \\
\hline miR-372 & & & & 0.03 & & 0.05 & \\
\hline$\leqslant 1.02$ & 40 & 17 & 24 & & 1.00 (reference) & & 1.00 (reference) \\
\hline E2F1 & & & & 0.01 & & 0.01 & \\
\hline$\leqslant-1.72$ & 42 & 19 & 16 & & 1.00 (reference) & & 1.00 (reference) \\
\hline$>-1.72$ & 38 & 24 & 8 & & $1.96(1.18,3.27)$ & & $1.95(1.14,3.33)$ \\
\hline
\end{tabular}

Table 3. Evaluation of miR-21, miR-372, E2F1, and the combination of all three, on PFS in univariate and multivariate analyses

\begin{tabular}{|l|c|c|c|c|c|c|}
\cline { 2 - 6 } & \multicolumn{5}{c|}{ Univariate HR and 95\% Cl } & \multicolumn{2}{c|}{ Multivariate HR and 95\% Cl } \\
\cline { 2 - 7 } & P-value & $<$ Median & $>$ Median & $P$-value & $<$ Median & $>$ Median \\
\hline miR-21 & 0.009 & 1 (reference) & $1.97(1.18,3.30)$ & 0.01 & 1 (reference) & $2.01(1.17,3.45)$ \\
\hline miR-372 & 0.03 & 1 (reference) & $1.73(1.04,2.89)$ & 0.05 & 1 (reference) & $1.70(1.00,2.89)$ \\
\hline E2F1 & 0.01 & 1 (reference) & $1.96(1.18,3.27)$ & 0.01 & 1 (reference) & $1.95(1.14,3.33)$ \\
\hline miR-21, miR-372 and E2F1 & 0.0004 & 1 (reference) & $7.62(2.46,23.58)$ & 0.004 & 1 (reference) & $6.7(1.84,24.5)$ \\
\hline Abbreviations: Cl= confidence interval; HR= hazard ratio; PFS = progression-free survival. & & & \\
\hline
\end{tabular}

between the PFS groups adjusting for ECOG-PS status and presence of visceral disease. We determined that increased levels of miR-21 and miR-372 were the only statistically significant miRNAs associated with poor PFS $(P$-value $<0.05)$ on univariate and multivariate analyses. The dCt median for each miR was utilised as a dichotic threshold, and the associated statistical significance with respect to PFS are shown in Table 2. Higher E2F1 - above the median - was the only TF statistically associated with PFS $(P$-value $<0.05)$, with higher levels conferring poorer outcomes. The significant association of E2F1 with PFS in both univariate and multivariable analyses adjusting for ECOG status and presence of visceral disease is shown in Table 2.

When combining all three significant features, increased levels of E2F1 $(P=0.01$, HR: $1.95(1.14,3.33))$, and high miR-21 $(P=0.01$, HR: $2.01(1.17,3.45))$ and miR-372 $(P=0.05$, HR: 1.70 $(1.00,2.89))$, were associated with a shorter PFS. Patients with altered expression in all three molecular factors had a median PFS of 2.5 months, while the median PFS for those with none of these alterations had not been reached. Table 3 shows the role of miR-21, miR-372 and the E2F1 on PFS in univariate and multivariate analyses. The combination of high miR-21, miR-372 and E2F1 was robustly associated with PFS on multivariate analysis $(P=0.004$, $\mathrm{HR}=6.7(1.84,24.5))$.

In the analysis of the association of miR-372, miR-21 and E2F1 with the extreme or outlier phenotype of PFS (progressed or died within 4 months $v s>$ alive without progression at 12 months), only miR-21 and E2F1 were marginally statistically significant (Table 4). A PFS curve based on high tumour miRNA levels of miR-21 (A), miR-372 (B), E2F1 (C) and combination of miR-21 and E2F1 (D) is shown in Figure 1A-D.

An exploratory multivariate analysis for association of OS showed a trend of higher expression level of miR-21 $(\mathrm{HR}=1.75$ with 95\% CI: $1.00-3.09, P=0.05)$ and for E2F1 (HR $=1.57$ with 95\% CI: $0.89-2.77, P=0.12$ ) associated with higher risk of death (data not shown).

Association of clinical variables with PFS. We included the type of treatment (M-VAC vs Cisplatin Gemcitabine) as a covariate in
Table 4. Association of E2F1, miR-372 and miR-21 with the extreme or outlier phenotype of PFS (progressed or died within 4 months vs $>$ alive without progression at 12 months)

\begin{tabular}{|c|c|c|c|}
\hline & $\mathbf{N}$ & Median (q1, q3) & $P$-value \\
\hline E2F1 & & & 0.055 \\
\hline Progressed or died within 4 months & 8 & $-1.19(-1.69,-0.64)$ & \\
\hline Alive without progression at 12 months & 29 & $-1.98(-2.59,-1.50)$ & \\
\hline miR-372 & & & 0.11 \\
\hline Progressed or died within 4 months & 8 & $1.54(1.00,1.70)$ & \\
\hline Alive without progression at 12 months & 29 & $0.78(0.22,1.44)$ & \\
\hline $\operatorname{miR}-21$ & & & 0.05 \\
\hline Progressed or died within 4 months & 8 & $0.56(-0.24,1.27)$ & \\
\hline Alive without progression at 12 months & 29 & $-0.25(-0.53,0.38)$ & \\
\hline
\end{tabular}

multivariable analysis, the associations of markers with outcome remained and we did not observe a trend of MVAC improving PFS (HR: 1.17 with 95\% CI: 0.69, 1.99).

Exploratory analysis in TCGA. Levels of E2F1 have been associated with prognosis in bladder cancer also with an aggregate signature with EZH2 and SUZ2. The outcome analysis of E2F1, EZH2 and SUZ12 expression in TCGA showed that these 3 genes are highly upregulated in tumours compared with 15 normal samples confirming its implication in bladder cancers. Besides, all 3 gene expressions were strongly correlated each other. The Pearson correlation between E2F1 and EZH2 was 0.66 and between E2F1 and SUZ12 was 0.41. However, with the short follow-up in TCGA being a significant confounding factor, the association of these three genes with outcome was not consistent with each other.

To follow, we looked for the association of EZH2 and outcome, in our patients. An association was observed, but when we 
A

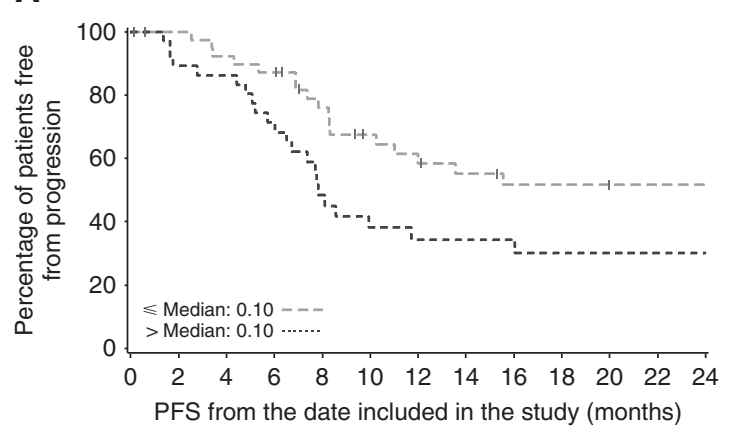

B

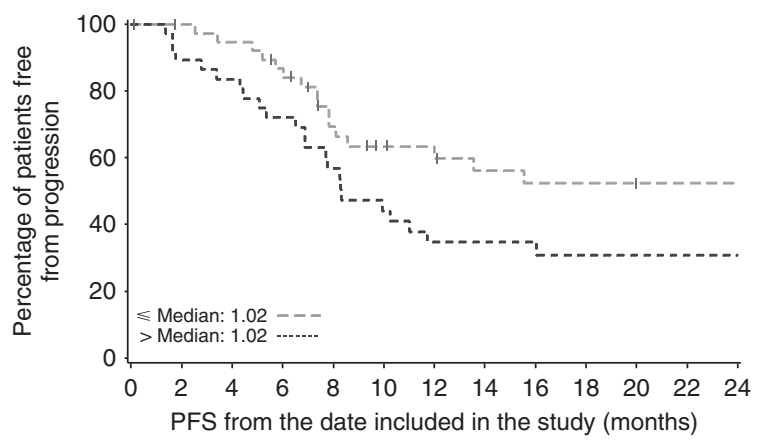

C

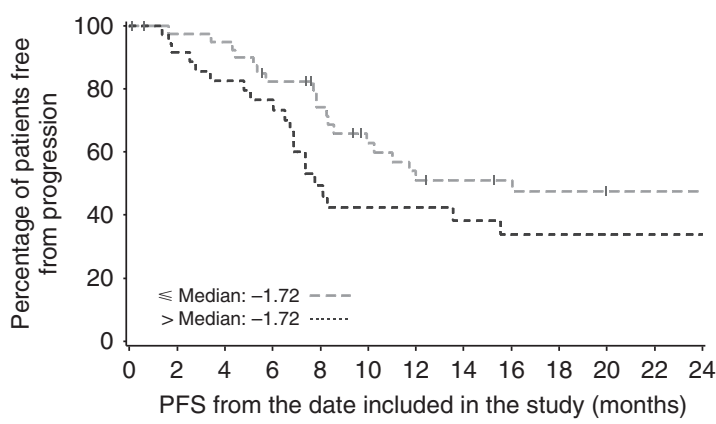

D

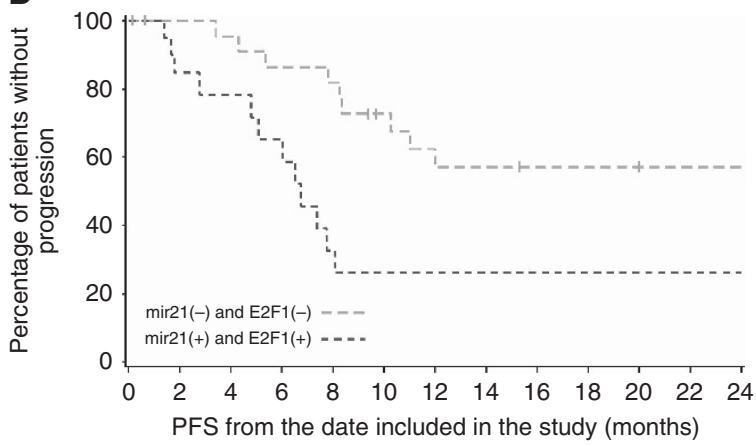

Figure 1. Progression-free survival (PFS) based on high tumour miRNA levels of miR-21 (A), miR-372 (B), E2F1 (C) and combination of miR-21 and E2F1 (D).

included EZH2, E2F1 and miR-21 in the model, only E2F1 and miR-21 remained significant (data not shown).

The association of the markers studied with molecular subtypes was also addressed. miR-21 was a differentially expressed maker, but it was downregulated in mRNA cluster II (luminal). No analysis was available with E2F1, EZH2 or SUZ2 in terms of mRNA subtypes.

Finally, no platinum therapy data is available to correlate with miR data in bladder TCGA, since the tissue analysed is obtained from cystectomies and median follow-up is short.

Activity of cisplatin in UC cell lines. A range of inhibitory concentrations $(\mathrm{GI})_{50}$ were observed when we investigated the activity of cisplatin in eight UC cell lines (Figure 2).

There was no correlation between miR-21 expression and $\mathrm{GI}_{50}$ (Supplementary Figure 1). In cell lines with relatively reduced expression of miR-21 (RT-4, RT112, 647-V and VM-CUB1), exogenous expression by lentiviral transduction resulted in increased levels of miR-21 as measured by qRT-PCR relative to transduction with a control precursor miRNA (Supplementary Figure 2A). However, there was no subsequent effect on cisplatin sensitivity (Supplementary Figures $2 \mathrm{~B}$ and 3 ). In an analogous fashion, suppression of miR-21 by transduction with a lentivirus expressing antisense to miR-21 in cell lines with the highest miR21 expression (J82, T24, TCC-SUP and UMUC3) did not alter cisplatin sensitivity (Supplementary Figure 3).

\section{DISCUSSION}

In our study of metastatic UC patients treated with cisplatin-based therapy, tumour levels of E2F1 gene expression, and miR-21, and miR-372 correlated with PFS independent of clinical prognostic factors, including visceral metastasis and ECOG-PS. miRs have been known to be differentially expressed in UC tissue and they have been correlated with cancer development. However, the mechanism of action of regulation of cancer development or response to treatment is poorly understood. Certain miRs have

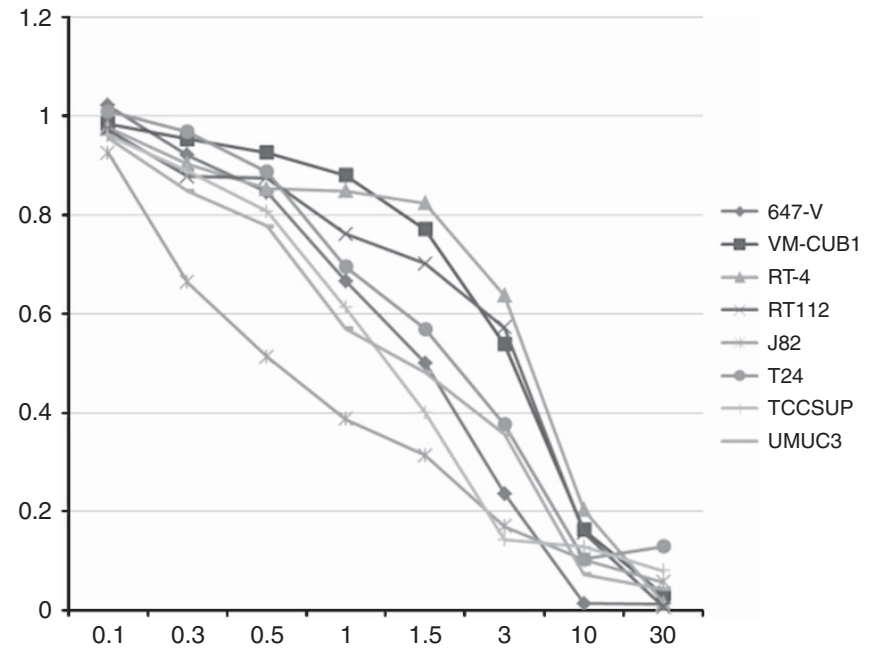

Figure 2. Sensitivity of bladder cancer cell lines to cisplatin in vitro.

been shown to act as oncogenes and tumour suppressors. Each $\mathrm{miR}$ is thought to target multiple mRNAs and each mRNA has multiple miRs that target it. Owing to these duplications, it is often difficult to identify a correlative relationship between the expression of miRs and mRNAs. On the basis of the available literature data, miR-21 is a relevant biological factor in multiple malignancies and has a potential role in predicting chemosensitivity (Saito et al, 2011; Wei et al, 2011; Akagi et al, 2013). Consequently, we specifically focused on its role in predicting outcomes in a clinical cohort of metastatic UC patients receiving platinum and in sensitivity to cisplatin in vitro in bladder cancer cell lines.

miR-21 has been shown to be upregulated in cancerous tissue, and thought to be an oncogene. For example, it appears upregulated during damage from radiation and helps repress Cdc25A, which is involved in cell cycle control (de Oliveira et al, 2009). High levels of miR-21 have been extensively reported in 
multiple studies to be associated with outcomes in UC as well as in other malignancies (Zhou et al, 2014). A recent focused metaanalysis from 19 bladder cancer data sets identified three upregulated (miR-141, miR-200c and miR-21) and five downregulated (miR-145, miR-125, miR-199a, let-7c and miR-99a) miRs associated with OS (Zhou et al, 2015). Increased expression of miR-21 has also been shown to confer resistance to platinum in preclinical systems and appears prognostic in the post-operative setting of non-small-cell lung cancer (Saito et al, 2011; Wei et al, 2011; Akagi et al, 2013). Thus, our observation of the association of high miR-21 with shorter survival is consistent with these previous reports. However, the sensitivity of UC cell lines to cisplatin in vitro did not vary based on miR-21 levels, suggesting that miR levels are prognostic but not predictive in the setting of cisplatin-based chemotherapy for UC. Similar data exist for prognostic impact of intrinsic subtypes (e.g., luminal, basal and p53 type) of UC based on gene expression data and on the PD-L1 immunohistochemical expression (Bellmunt et al, 2015), although the predictive impact of these classifications is unclear (Sjodahl et al, 2012; Cancer Genome Atlas Research Network, 2014; Choi et al, 2014; Damrauer et al, 2014). Emerging data also support the predictive impact of alterations in DNA repair genes ( $A T M, R B 1, F A N C C, E R C C 2$ or ERBB2) on response to neoadjuvant platinum-based chemotherapy (Rosenberg et al, 2014; Groenendijk et al, 2015; McConkey et al, 2015; Plimack et al, 2015). Interestingly, we have observed that miR-21 is found to be downregulated in mRNA cluster II (luminal) suggesting its adverse prognostic role.

miR-372, another of the miRs identified to be prognostic in our study, has been previously shown to be involved in the epithelial-mesenchymal transition (EMT), downregulation of cyclin-dependent kinase (CDK)-2 and epigenetic regulation (Subramanyam et al, 2011; Tian et al, 2011). One of the mechanisms of activity of miR-372 appears to be the inhibition of transforming growth factor- $\beta$-induced EMT of human epithelial cells. miR-372 could be a non-invasive biomarker for prognosis assessment in several tumour types (Tan et al, 2014; Tu et al, 2015; Yu et al, 2015). For example, in colorectal cancer a distinct miR expression signature on serum miRNA372 has been identified as a biomarker predicting chemosensitivity (Zhang et al, 2014).

Among TFs, we selected to study E2F1, TWIST and RELA due to their clinical significance in UC. Consistent with other cancers (Alla et al, 2010; Gu et al, 2014), we observed a relationship between cancer progression and the overexpression of E2F1 gene. Of note, in patients with non-muscle invasive bladder cancer, increased tumour recurrence and progression has been associated with increased E2F expression (Santos et al, 2014). Also, transcriptional changes of E2F1 with its upstream counterparts EZH2, and SUZ12 predict bladder cancer aggressiveness, as well as anticancer drug resistance (Lee et al, 2015). While the E2F1 interaction with hsa-miR-21-5p has been validated, the recent discovery of EZH2 having a relevant role in bladder cancer is biologically relevant to urothelial carcinogenesis. It has been experimentally demonstrated that $\mathrm{EZH} 2$ and $\mathrm{E} 2 \mathrm{~F} 1$ form a transcriptional complex co-localising at specific chromatin sites to activate the downstream target genes. The mechanisms underlying EZH2-E2F1 complex for cancer progression and their therapeutic potential await future studies. The exploratory analysis in our cohort did not find an association between EZH2 and outcome without being able to analyse its role on progression.

Our study is limited by a modest sample size of 83 patients and the retrospective design. Second, it mainly addresses a clinical/ tissue correlation and the chemosensitivity was only tested in vitro. Also, the analysis was done in samples from primary tumours and not from metastatic sites. The panel of miRs studied may not have included some significant miRs, which have been reported across other studies, for example, we did not include miR-129, which was found to be associated with better prognosis in a study of 106 bladder tumour samples (Dyrskjot et al, 2009), or miRNA-27a associated with resistance in bladder cell lines (Drayton et al, 2014). In addition, the modest size of our data set may have prevented us from detecting the significance of some miRs, for example, miR-106b and miR-10b were found to be univariately associated with outlying responses, but were not significant on overall PFS analysis or multivariate analysis. Similarly, TWIST1 was significantly associated with outlying poor PFS on univariable analyses, but not in a TTF analysis of the whole cohort, which may be a function of sample size. Indeed, miR-106b, miR-10b and TWIST1 have all been reported to exert prognostic impacts in other studies (Sampath et al, 2009; Fritz et al, 2014; Wang et al, 2015). Finally, the dichotomisation of using the median for the values is also a potential limitation and, perhaps another cut-point may be more biologically and prognostically relevant.

To conclude, high tumour tissue miR-21 and low E2F1 conferred a significant negative impact on PFS in patients with advanced UC receiving first-line cisplatin-based chemotherapy. Given the small size and robustness of this molecule, miR can be readily measured in archival FFPE tumour as well as plasma samples. Unfortunately, our preclinical data could not confirm the value of miR-21 in predicting sensitivity to cisplatin in vitro.

Taking into account all of these limitations, our analysis is descriptive and exploratory and is unlikely to provide immediate clinical benefit as a biomarkers. External validation with statistical power, combining mir-21 with clinical variables known to have a prognostic role in bladder cancer is the next step to follow. We suggest that mir-21 may be included as a biological prognostic factor in future studies.

Nevertheless, study of a larger panel of miRs including those identified by us in a larger data set could provide an improved molecular prognostic risk grouping, and potentially other miRs predictive of benefit. Potentially, panels of TFs and miRs coupled with other alterations at the gene level will improve the ability to develop tailored therapy. A composite prognostic model consisting of currently used clinical factors and a comprehensive molecular panel accounting for the tumour and microenvironment will enhance the interpretation of trials and progress towards the goal precision medicine. Indeed, preliminary data have been reported for the prognostic impact of a combination of clinicopathologic factors and gene expression profile following RC for bladder cancer (Riester et al, 2012).

\section{ACKNOWLEDGEMENTS}

This work has been supported by FIS PI10/02112 (microRNAS) (Spanish Health Ministry Grant 'Fondo de Investigacion Sanitaria') and RTICC 06/0020/19 grants. Fundació Cellex (Barcelona) provided a generous donation to the Group of Molecular Therapeutics and Biomarkers, Hospital del Mar. This study was also possible thanks to a grant from Friends of Dana-Farber, the Retired Professional Fire Fighters Cancer Fund, and the generous support of Whole Foods Golf Classic for bladder cancer research in memoriam of Christopher Snell. Also a private donation in the memory of Rich Beaudoin. The funders had no role in study design, data collection and analysis, decision to publish, or preparation of the manuscript.

\section{CONFLICT OF INTEREST}

JB: consultant for Pierre Fabre, Astellas, Pfizer, Merck and Genentech. Research funding from Takeda and Sanofi. GS: research support from Onyx, Bayer and Boehringer-Ingelheim; 
advisory board/consultant for Merck, Genentech, Sanofi, Bayer, Pfizer and Novartis. The remaining authors declare no conflict of interest.

\section{REFERENCES}

Akagi I, Okayama H, Schetter AJ, Robles AI, Kohno T, Bowman ED, Kazandjian D, Welsh JA, Oue N, Saito M, Miyashita M, Uchida E, Takizawa T, Takenoshita S, Skaug V, Mollerup S, Haugen A, Yokota J, Harris CC (2013) Combination of protein coding and noncoding gene expression as a robust prognostic classifier in stage I lung adenocarcinoma. Cancer Res 73(13): 3821-3832.

Alla V, Engelmann D, Niemetz A, Pahnke J, Schmidt A, Kunz M, Emmrich S, Steder M, Koczan D, Putzer BM (2010) E2F1 in melanoma progression and metastasis. J Natl Cancer Inst 102(2): 127-133.

Apolo AB, Ostrovnaya I, Halabi S, Iasonos A, Philips GK, Rosenberg JE, Riches J, Small EJ, Milowsky MI, Bajorin DF (2013) Prognostic model for predicting survival of patients with metastatic urothelial cancer treated with cisplatin-based chemotherapy. J Natl Cancer Inst 105(7): 499-503.

Bajorin DF, Dodd PM, Mazumdar M, Fazzari M, McCaffrey JA, Scher HI, Herr H, Higgins G, Boyle MG (1999) Long-term survival in metastatic transitional-cell carcinoma and prognostic factors predicting outcome of therapy. J Clin Oncol 17(10): 3173-3181.

Bartel DP (2004) MicroRNAs: genomics, biogenesis, mechanism, and function. Cell 116(2): 281-297.

Bellmunt J, Mullane SA, Werner L, Fay AP, Callea M, Leow JJ, Taplin ME, Choueiri TK, Hodi FS, Freeman GJ, Signoretti S (2015) Association of PD-L1 expression on tumor-infiltrating mononuclear cells and overall survival in patients with urothelial carcinoma. Ann Oncol 26(4): 812-817.

Choi W, Porten S, Kim S, Willis D, Plimack ER, Hoffman-Censits J, Roth B, Cheng T, Tran M, Lee IL, Melquist J, Bondaruk J, Majewski T, Zhang S, Pretzsch S, Baggerly K, Siefker-Radtke A, Czerniak B, Dinney CP, McConkey DJ (2014) Identification of distinct Basal and luminal subtypes of muscle-invasive bladder cancer with different sensitivities to frontline chemotherapy. Cancer Cell 25(2): 152-165.

Cancer Genome Atlas Research Network. Comprehensive molecular characterization of urothelial bladder carcinoma (2014) Nature 507(7492): 315-322.

Damrauer JS, Hoadley KA, Chism DD, Fan C, Tiganelli CJ, Wobker SE, Yeh JJ, Milowsky MI, Iyer G, Parker JS, Kim WY (2014) Intrinsic subtypes of high-grade bladder cancer reflect the hallmarks of breast cancer biology. Proc Natl Acad Sci USA 111(8): 3110-3115.

de Oliveira PE, Zhang L, Wang Z, Lazo JS (2009) Hypoxia-mediated regulation of Cdc25A phosphatase by p21 and miR-21. Cell Cycle 8(19): 3157-3164.

Dijkstra JR, Mekenkamp LJ, Teerenstra S, De Krijger I, Nagtegaal ID (2012) MicroRNA expression in formalin-fixed paraffin embedded tissue using real time quantitative PCR: the strengths and pitfalls. J Cell Mol Med 16(4): 683-690.

Drayton RM, Dudziec E, Peter S, Bertz S, Hartmann A, Bryant HE, Catto JW (2014) Reduced expression of miRNA-27a modulates cisplatin resistance in bladder cancer by targeting the cystine/glutamate exchanger SLC7A11. Clin Cancer Res 20(7): 1990-2000.

Dyrskjot L, Ostenfeld MS, Bramsen JB, Silahtaroglu AN, Lamy P, Ramanathan R, Fristrup N, Jensen JL, Andersen CL, Zieger K, Kauppinen S, Ulhoi BP, Kjems J, Borre M, Orntoft TF (2009) Genomic profiling of microRNAs in bladder cancer: miR-129 is associated with poor outcome and promotes cell death in vitro. Cancer Res 69(11): 4851-4860.

Fritz HK, Lindgren D, Ljungberg B, Axelson H, Dahlback B (2014) The $\operatorname{miR}(21 / 10 \mathrm{~b})$ ratio as a prognostic marker in clear cell renal cell carcinoma. Eur J Cancer 50(10): 1758-1765.

Groenendijk FH, de Jong J, Fransen van de Putte EE, Michaut M, Schlicker A, Peters D, Velds A, Nieuwland M, van den Heuvel MM, Kerkhoven RM, Wessels LF, Broeks A, van Rhijn BW, Bernards R, van der Heijden MS (2015) ERBB2 mutations characterize a subgroup of muscle-invasive bladder cancers with excellent response to neoadjuvant chemotherapy. Eur Urol 69(3): 384-388.

Gu Y, Cheng Y, Song Y, Zhang Z, Deng M, Wang C, Zheng G, He Z (2014) MicroRNA-493 suppresses tumor growth, invasion and metastasis of lung cancer by regulating E2F1. PLoS One 9(8): e102602.
Guancial EA, Bellmunt J, Yeh S, Rosenberg JE, Berman DM (2014) The evolving understanding of microRNA in bladder cancer. Urol Oncol 32(1): 41 e31-40.

Guo H, Ingolia NT, Weissman JS, Bartel DP (2010) Mammalian microRNAs predominantly act to decrease target mRNA levels. Nature 466(7308): 835-840.

Lee SR, Roh YG, Kim SK, Lee JS, Seol SY, Lee HH, Kim WT, Kim WJ, Heo J, Cha HJ, Kang TH, Chung JW, Chu IS, Leem SH (2015) Activation of EZH2 and SUZ12 regulated by E2F1 predicts the disease progression and aggressive characteristics of bladder cancer. Clin Cancer Res 21(23): 5391-5403.

McConkey DJ, Choi W, Shen Y, Lee IL, Porten S, Matin SF, Kamat AM, Corn P, Millikan RE, Dinney C, Czerniak B, Siefker-Radtke AO (2015) A prognostic gene expression signature in the molecular classification of chemotherapy-naive urothelial cancer is predictive of clinical outcomes from neoadjuvant chemotherapy: a phase 2 trial of dose-dense methotrexate, vinblastine, doxorubicin, and cisplatin with bevacizumab in urothelial cancer. Eur Urol; e-pub ahead of print 2 September 2015; doi:10.1016/j.eururo.2015.08.034.

Mukherjee N, Houston TJ, Cardenas E, Ghosh R (2015) To be an ally or an adversary in bladder cancer: the NF-kappaB story has not unfolded. Carcinogenesis 36(3): 299-306.

Plimack ER, Dunbrack RL, Brennan TA, Andrake MD, Zhou Y, Serebriiskii IG, Slifker M, Alpaugh K, Dulaimi E, Palma N, Hoffman-Censits J, Bilusic M, Wong YN, Kutikov A, Viterbo R, Greenberg RE, Chen DY, Lallas CD, Trabulsi EJ, Yelensky R, McConkey DJ, Miller VA, Golemis EA, Ross EA (2015) Defects in DNA repair genes predict response to neoadjuvant cisplatin-based chemotherapy in muscle-invasive bladder cancer. Eur Urol 68(6): 959-967.

Riester M, Taylor JM, Feifer A, Koppie T, Rosenberg JE, Downey RJ, Bochner BH, Michor F (2012) Combination of a novel gene expression signature with a clinical nomogram improves the prediction of survival in high-risk bladder cancer. Clin Cancer Res 18(5): 1323-1333.

Rosenberg JE, Van Allen EM, Mouw KW, Kim PH, Wagle N, Al-Ahmadie H, Zhu C, Ostrovnaya I, Iyer G, Signoretti S, Reuter VE, Getz G, Kantoff PW, Bochner BH, Choueiri TK, Bajorin DF, Solit DB, Gabriel SB, D'Andrea AD, Garraway LA (2014) Association of somatic ERCC2 mutations with cisplatin sensitivity in muscle-invasive urothelial carcinoma. J Clin Oncol 32: 5 s(suppl): abstr 4510 .

Saito M, Schetter AJ, Mollerup S, Kohno T, Skaug V, Bowman ED, Mathe EA, Takenoshita S, Yokota J, Haugen A, Harris CC (2011) The association of microRNA expression with prognosis and progression in early-stage, nonsmall cell lung adenocarcinoma: a retrospective analysis of three cohorts. Clin Cancer Res 17(7): 1875-1882.

Sampath D, Calin GA, Puduvalli VK, Gopisetty G, Taccioli C, Liu CG, Ewald B, Liu C, Keating MJ, Plunkett W (2009) Specific activation of microRNA106b enables the p73 apoptotic response in chronic lymphocytic leukemia by targeting the ubiquitin ligase Itch for degradation. Blood 113(16): 3744-3753.

Santos M, Martinez-Fernandez M, Duenas M, Garcia-Escudero R, Alfaya B, Villacampa F, Saiz-Ladera C, Costa C, Oteo M, Duarte J, Martinez V, Gomez-Rodriguez MJ, Martin ML, Fernandez M, Viatour P, Morcillo MA, Sage J, Castellano D, Rodriguez-Peralto JL, de la Rosa F, Paramio JM (2014) In vivo disruption of an Rb-E2F-Ezh2 signaling loop causes bladder cancer. Cancer Res 74(22): 6565-6577.

Siegel RL, Miller KD, Jemal A (2015) Cancer statistics, 2015. CA Cancer J Clin 65(1): 5-29.

Sjodahl G, Lauss M, Lovgren K, Chebil G, Gudjonsson S, Veerla S, Patschan O, Aine M, Ferno M, Ringner M, Mansson W, Liedberg F, Lindgren D, Hoglund M (2012) A molecular taxonomy for urothelial carcinoma. Clin Cancer Res 18(12): 3377-3386.

Sonpavde G, Bellmunt J (2015) Salvage systemic therapy for advanced urothelial carcinoma: on the cusp of a sea change? Oncologist 20(5): 461-463.

Subramanyam D, Lamouille S, Judson RL, Liu JY, Bucay N, Derynck R, Blelloch R (2011) Multiple targets of miR-302 and miR-372 promote reprogramming of human fibroblasts to induced pluripotent stem cells. Nat Biotechnol 29(5): 443-448.

Tan JK, Tan EL, Gan SY (2014) Elucidating the roles of miR-372 in cell proliferation and apoptosis of nasopharyngeal carcinoma TW01 cells. Exp Oncol 36(3): 170-173.

Tian RQ, Wang XH, Hou LJ, Jia WH, Yang Q, Li YX, Liu M, Li X, Tang H (2011) MicroRNA-372 is down-regulated and targets cyclin-dependent 
kinase 2 (CDK2) and cyclin A1 in human cervical cancer, which may contribute to tumorigenesis. J Biol Chem 286(29): 25556-25563.

Tu HF, Chang KW, Cheng HW, Liu CJ (2015) Upregulation of miR-372 and -373 associates with lymph node metastasis and poor prognosis of oral carcinomas. Laryngoscope 125: E365-E370.

von der Maase H, Sengelov L, Roberts JT, Ricci S, Dogliotti L, Oliver T, Moore MJ, Zimmermann A, Arning M (2005) Long-term survival results of a randomized trial comparing gemcitabine plus cisplatin, with methotrexate, vinblastine, doxorubicin, plus cisplatin in patients with bladder cancer. J Clin Oncol 23(21): 4602-4608.

Wang N, Guo D, Zhao Y, Dong C, Liu X, Yang B, Wang S, Wang L, Liu Q, Ren Q, Lin Y, Ma X (2015) TWIST-1 promotes cell growth, drug resistance and progenitor clonogenic capacities in myeloid leukemia and is a novel poor prognostic factor in acute myeloid leukemia. Oncotarget 6(25): 20977-20992.

Wei J, Gao W, Zhu CJ, Liu YQ, Mei Z, Cheng T, Shu YQ (2011) Identification of plasma microRNA-21 as a biomarker for early detection and chemosensitivity of non-small cell lung cancer. Chin J Cancer 30(6): 407-414.

Wushou A, Hou J, Zhao YJ, Shao ZM (2014) Twist-1 up-regulation in carcinoma correlates to poor survival. Int J Mol Sci 15(12): 21621-21630.

Yu J, Jin L, Li W, Jiang L, Hu Y, Zhi Q, Zhu X (2015) Serum miR-372 is a diagnostic and prognostic biomarker in patients with early colorectal cancer. Anticancer Agents Med Chem 16(4): 424-431.

Yu SL, Chen HY, Chang GC, Chen CY, Chen HW, Singh S, Cheng CL, Yu CJ, Lee YC, Chen HS, Su TJ, Chiang CC, Li HN, Hong QS, Su HY, Chen CC, Chen WJ, Liu CC, Chan WK, Chen WJ, Li KC, Chen JJ, Yang PC (2008)
MicroRNA signature predicts survival and relapse in lung cancer. Cancer Cell 13(1): 48-57.

Zhang J, Zhang K, Bi M, Jiao X, Zhang D, Dong Q (2014) Circulating microRNA expressions in colorectal cancer as predictors of response to chemotherapy. Anticancer Drugs 25(3): 346-352.

Zhang L, Volinia S, Bonome T, Calin GA, Greshock J, Yang N, Liu CG, Giannakakis A, Alexiou P, Hasegawa K, Johnstone CN, Megraw MS, Adams S, Lassus H, Huang J, Kaur S, Liang S, Sethupathy P, Leminen A, Simossis VA, Sandaltzopoulos R, Naomoto Y, Katsaros D, Gimotty PA, DeMichele A, Huang Q, Butzow R, Rustgi AK, Weber BL, Birrer MJ, Hatzigeorgiou AG, Croce CM, Coukos G (2008) Genomic and epigenetic alterations deregulate microRNA expression in human epithelial ovarian cancer. Proc Natl Acad Sci USA 105(19): 7004-7009.

Zhou H, Tang K, Xiao H, Zeng J, Guan W, Guo X, Xu H, Ye Z (2015) A panel of eight-miRNA signature as a potential biomarker for predicting survival in bladder cancer. J Exp Clin Cancer Res 34: 53.

Zhou X, Wang X, Huang Z, Wang J, Zhu W, Shu Y, Liu P (2014) Prognostic value of miR-21 in various cancers: an updating meta-analysis. PLoS One 9(7): e102413.

This work is published under the standard license to publish agreement. After 12 months the work will become freely available and the license terms will switch to a Creative Commons AttributionNonCommercial-Share Alike 4.0 Unported License.

Supplementary Information accompanies this paper on British Journal of Cancer website (http://www.nature.com/bjc) 